Bas J. W. Dekkers · Jolanda A. M. J. Schuurmans

Sjef C. M. Smeekens

\title{
Glucose delays seed germination in Arabidopsis thaliana
}

Received: 7 May 2003/ Accepted: 13 October 2003/Published online: 25 November 2003

(C) Springer-Verlag 2003

\begin{abstract}
Here we report that glucose delays germination of Arabidopsis thaliana (L.) Heynh. seeds at concentrations below those known to inhibit early seedling development. This inhibition acts on embryo growth and is independent of hexokinase (HXK) function. Hormones and hormone inhibitors were applied to the germination media and several hormone biosynthesis and signalling mutants were tested on glucose media to investigate a possible role of abscisic acid (ABA), gibberellin and ethylene in the glucose-induced germination delay. Results indicate that the germination inhibition by glucose cannot be antagonized by ethylene or gibberellin and is independent of the $H X K 1 / \mathrm{ABA} / A B I 4$ signalling cascade. These findings suggest that there is a separate regulatory pathway independent of $A B I 2 /$ $A B I 4 / A B I 5$. Thus, in a relatively short time frame sugars utilize different signalling cascades to inhibit germination and post-germination growth, underlining the complexity of sugar responses.
\end{abstract}

Keywords Abscisic acid - Arabidopsis - Ethylene · Germination · Gibberellic acid · Glucose signalling

Abbreviations $A B A$ Abscisic acid $\cdot A B I$ ABA insensitive $A C C$ 1-Aminocyclopropane-1-carboxylic acid $\cdot B R$ Brassinosteroid $\cdot C A B$ Chlorophyll $a / b$ binding protein $\cdot F U S 3$ Fusca3 $\cdot G A$ Gibberellin $\cdot G A_{3}$ Gibberellic acid $\cdot H X K$ Hexokinase $\cdot$ LEC1 Leafy cotyledon1 $\cdot R B C S$ Ribulose-1,5-bisphosphate carboxylase small subunit $\cdot W T$ Wild type

B. J. W. Dekkers $(\bowtie) \cdot$ J. A. M. J. Schuurmans

S. C. M. Smeekens

Department of Plant Biology,

Molecular Plant Physiology,

University of Utrecht, Padualaan 8,

$3584 \mathrm{CH}$ Utrecht,

The Netherlands

E-mail: s.j.w.dekkers@bio.uu.nl

Fax: + 31-30-2513655

\section{Introduction}

Photosynthesis provides plants with sugars that have a pivotal role in the plant life cycle. Carbohydrates serve as energy source and as basic building materials for the synthesis of essentially all other organic molecules. Furthermore, sugars can be converted into polymers giving rise to storage components like starch and fructans or structural components like cellulose. Sugars also regulate the expression of numerous genes (Koch 1996) and affect many important plant processes (Gibson 2000). Moreover, sugars have a signalling function in which the hexokinase (HXK) protein is suggested to play a pivotal role (Sheen et al. 1999; Smeekens 2000; Moore et al. 2003).

At least three different glucose-signalling pathways have been proposed in plants (Sheen et al. 1999). An HXK-independent pathway regulates genes like those for chalcone synthase $(C H S)$ and cell wall invertase 1 (CIN1). These genes are induced by sugar analogs such as 6-deoxyglucose (6-DG) and 3-O-methyl glucose (3-OMG) that are taken up by plant cells but not further metabolized. Such hexose sensing might be mediated by transporter-like receptors as described in yeast (Özcan et al. 1996), although these have not been demonstrated in plants so far. The two other pathways are HXKdependent, of which one is glycolysis-dependent and is induced by overexpression of both native Arabidopsis AtHXK1 and heterologous yeast HXK1 in Arabidopsis. The other pathway requires Arabidopsis AtHXK1 specifically and affects, among others, photosynthetic genes like those for chlorophyll $a / b$-binding protein (CAB), the small subunit of ribulose-1,5-bisphosphate carboxylase (RBCS) and plastocyanin (PC; Sheen et al. 1999; Moore et al. 2003).

To study plant-specific sugar signal transduction, several laboratories employed mutant screens in Arabidopsis and such screens identified so-called sugar insensitive mutants. Most of these screens revealed a central role for the plant hormones abscisic acid (ABA; 
Arenas-Huertero et al. 2000; Huijser et al. 2000; Laby et al. 2000; Rook et al. 2001) and ethylene (Zhou et al. 1998; Gibson et al. 2001) in sugar-induced signalling during early seedling development (for review, see Gazzarrini and McCourt 2001; Rolland et al. 2002). Many other processes are affected by sugars as well, including germination (Garciarrubio et al. 1997; Pego et al. 1999; Finkelstein and Lynch 2000).

Germination is a highly regulated process that is influenced by environmental factors, including light and temperature (Bentsink and Koornneef 2002). In addition, endogenous plant hormones play an important role in preventing (ABA) or stimulating [gibberellin (GA), brassinosteroid (BR) and ethylene] germination. Of these hormones, ABA and GA have the most pronounced effect. This is shown by the reduced seed dormancy of $a b a$ and several abi mutants (Koornneef et al. 1982, 1984), while the GA-deficient mutants (gal, ga2 and ga3) are incapable of germination (Debeaujon and Koornneef 2000; Bentsink and Koornneef 2002). Previous studies showed that mannose and 2-deoxyglucose, both of which are substrates for HXK, are potent inhibitors of Arabidopsis seed germination (Pego et al. 1999). Sugar uptake is probably not involved since the non-metabolizable sugars 6-DG and 3-OMG do not affect germination in this assay. The inhibiting effect of mannose could be suppressed by application of mannoheptulose, an inhibitor of HXK function, thereby suggesting HXK involvement. Interestingly, germination in mannose-arrested seeds could also be restored by addition of glucose to the medium. A similar effect of glucose was found for ABA-mediated inhibition of Arabidopsis seed germination. Application of metabolizable sugars relieved the inhibitory effect of ABA on germination but not on seedling growth (Garciarrubio et al. 1997; Finkelstein and Lynch 2000). The glucose relief of ABA action was already effective at a concentration of $35 \mathrm{mM}$ and the effect was enhanced by light (Finkelstein and Lynch 2000). Thus far, however, the mechanism by which metabolizable sugars are able to suppress the mannose and ABA inhibition of germination is still unresolved, although it does not seem to be solely nutritional (Finkelstein and Lynch 2000; Pritchard et al. 2003).

The studies described above suggest a stimulatory role for glucose in germination but, in contrast, when analyzing seed germination of several Arabidopsis ecotypes, we observed that even low concentrations of exogenously supplied sugars delayed seed germination. Here we report an analysis of this phenomenon at a physiological and genetic level. We conclude that this glucose response acts on the growth potential of the embryo, is not influenced by exogenous nitrogen availability, and is independent of HXK activity. To unravel a possible mechanism for the inhibition of germination by glucose we investigated the role of several plant hormones such as GAs, ethylene and ABA. These hormones play an important role in the control of seed germination and are involved in sugar responses as well. This analysis shows that ethylene and GA signalling are not able to antagonize the glucose-induced delay of seed germination. Furthermore, ABA levels affect the response to glucose during germination but the abi mutants respond like the wild type (WT) to the glucoseinduced delay.

\section{Materials and methods}

Plant material

Most of the germination assays were performed with the Arabidopsis thaliana (L.) Heynh. accessions Landsberg erecta (Ler; Lehle Seeds, Round Rock, TX, USA) and Col-0. Various Arabidopsis mutants with altered ABA, GA and ethylene biosynthesis or signalling were tested for the germination response on glucose. Plants were grown in a climate chamber at $22^{\circ} \mathrm{C}$ with $70 \%$ humidity and a $16 \mathrm{~h} / 8 \mathrm{~h}$ light/dark cycle (Sylvania GRO-LUX fluorescent lamps; Technische Unie, Utrecht). Seed batches that were compared in germination assays were grown simultaneously and harvested and stored under similar conditions. Seeds were dry-stored in paper bags for at least a month before use in germination experiments.

\section{Germination assays}

All germination assays were performed on $0.5 \mathrm{MS}$ : half-strength Murashige and Skoog medium (pH 5.8), including vitamins, solidified with $0.8 \%$ plant agar (Duchefa, Haarlem, The Netherlands). All sugars were obtained from Sigma-Aldrich except sucrose, which was obtained from Merck. Before plating, seeds were surface-sterilized in $20 \%(\mathrm{v} / \mathrm{v})$ commercial bleach (Glorix) for 8 10 min followed by 3-5 min in $70 \%$ (v/v) ethanol, and rinsed four to five times with sterile water. After a 3- to 4-day stratification period at $4^{\circ} \mathrm{C}$ in the dark, plates (with chemicals included as indicated) were incubated in a $16 \mathrm{~h} / 8 \mathrm{~h}$ light/dark cycle at $22^{\circ} \mathrm{C}$. Germination, scored by radicle emergence from the seed coat, was scored daily for 3-5 days. 'Single' experiments were performed in duplicate, each plate containing 50-100 seeds, and every duplicate experiment was repeated one to three times. For statistical analysis we calculated the confidence interval of the sample mean with the confidence tool of the Microsoft Excel package. It should be noted that during the time course of these experiments we observed that, in general, the germination rate was somewhat retarded compared to the initial observations. This was seen in different seed batches and accessions, which suggested that some external factor was responsible. This is surprising since the sterilization and plating procedures were standardized to limit seed germination variation. We tried to overcome this retardation by shifting plates to different places in the climate chamber and shortening the sterilization time of seeds. Furthermore, we started using completely fresh sterilization solutions and a new batch of MS salts, and stratified the seeds for an extra day, but this did not affect the general germination delay. Despite this retardation, similar trends in germination were observed in different batches.

\section{Preparation of Arabidopsis embryos}

Ler or Col-0 seeds were sterilized and imbibed for a few hours in sterile water. Embryos were isolated from the seeds using sterile forceps and scalpel. The isolated embryos were immediately plated on agar plates containing $0.5 \mathrm{MS}, 2.5 \%$ sorbitol or $2.5 \%$ glucose. The plates were stratified for 3-4 days before transfer to the growth chamber. Also, seeds were sown on the plates and seed germination was scored as a control for the glucose treatment. Embryo and 
seedling sizes were determined by taking photographs from which the sizes were scored. For each measurement 5-11 embryos were used.

\section{Results}

Exogenous glucose delays germination of Arabidopsis seeds

To investigate the effect of glucose on germination, seeds were plated on $0.5 \mathrm{MS}$ and a range $(0.5 \%, 1 \%$, $2.5 \%$ and $5 \%$ ) of glucose and sorbitol concentrations. Sorbitol is a sugar alcohol that is not taken up by plant cells and serves as an osmotic control (Gibson 2000). We observed that sugars delayed germination, as was previously reported for high glucose concentrations (6\% glucose) by To et al. (2002) and Ullah et al. (2002). The finding that even low concentrations (1\%) of glucose are able to delay seed germination (Fig. 1a) was unexpected, since low concentrations of metabolizable sugars are effective in relieving the inhibitory action of ABA and mannose during germination (Garciarrubio et al. 1997; Pego et al. 1999; Finkelstein and Lynch 2000). As expected, elevated levels of glucose (up to $5 \%$ ) restricted germination more severely. After 1 day the germination frequency in the sugar-free control plates approached $80-100 \%$. Since the media containing up to $2.5 \%$ sorbitol showed control-level germination frequencies, the germination delay is unlikely to have been caused by osmotic activity. The response to $2.5 \%$ sorbitol and glucose was studied in more detail. Germination was scored every $1.5-2.5 \mathrm{~h}$ from $16 \mathrm{~h}$ to $24 \mathrm{~h}$. The data presented in Fig. $1 \mathrm{~b}$ show that the germination frequency increases rapidly during the first day. Because of this fact we screened germination frequencies in as limited a time period as possible to prevent this time factor influencing the data scored. Germination on sorbitol is also slightly retarded but rapidly follows control-plate germination and the difference disappears after $24 \mathrm{~h}$.

Germination is both determined by growth potential of the embryo and the restrictive properties of the seed coat (Bentsink and Koornneef 2002; Debeaujon and Koornneef 2000). Isolated embryos from seeds were plated on $0.5 \mathrm{MS}$, and on $0.5 \mathrm{MS}$ containing $2.5 \%$ sorbitol or $2.5 \%$ glucose. However, removal of the seed coat did not neutralize the glucose effect, as shown by the fact that the glucose-treated seedlings were significantly smaller than the 0.5MS-treated embryos (Fig. 1c-f). This implies that sugar acts on the growth potential of the embryo directly. Moreover, the cotyledons of 0.5MS-treated embryos started greening after 1 day while the glucose-treated ones were white.

Thus far, we tested 10 different Arabidopsis accessions (Col-0, Ler, WS-2, C24, Oy1, Ksk1, Tsu, B-0, CVI and $\mathrm{Nd})$ for glucose $(1.5 \%)$ repression of germination. The accessions were grown simultaneously, except for Ler the seeds of which were 1 month older. All accessions
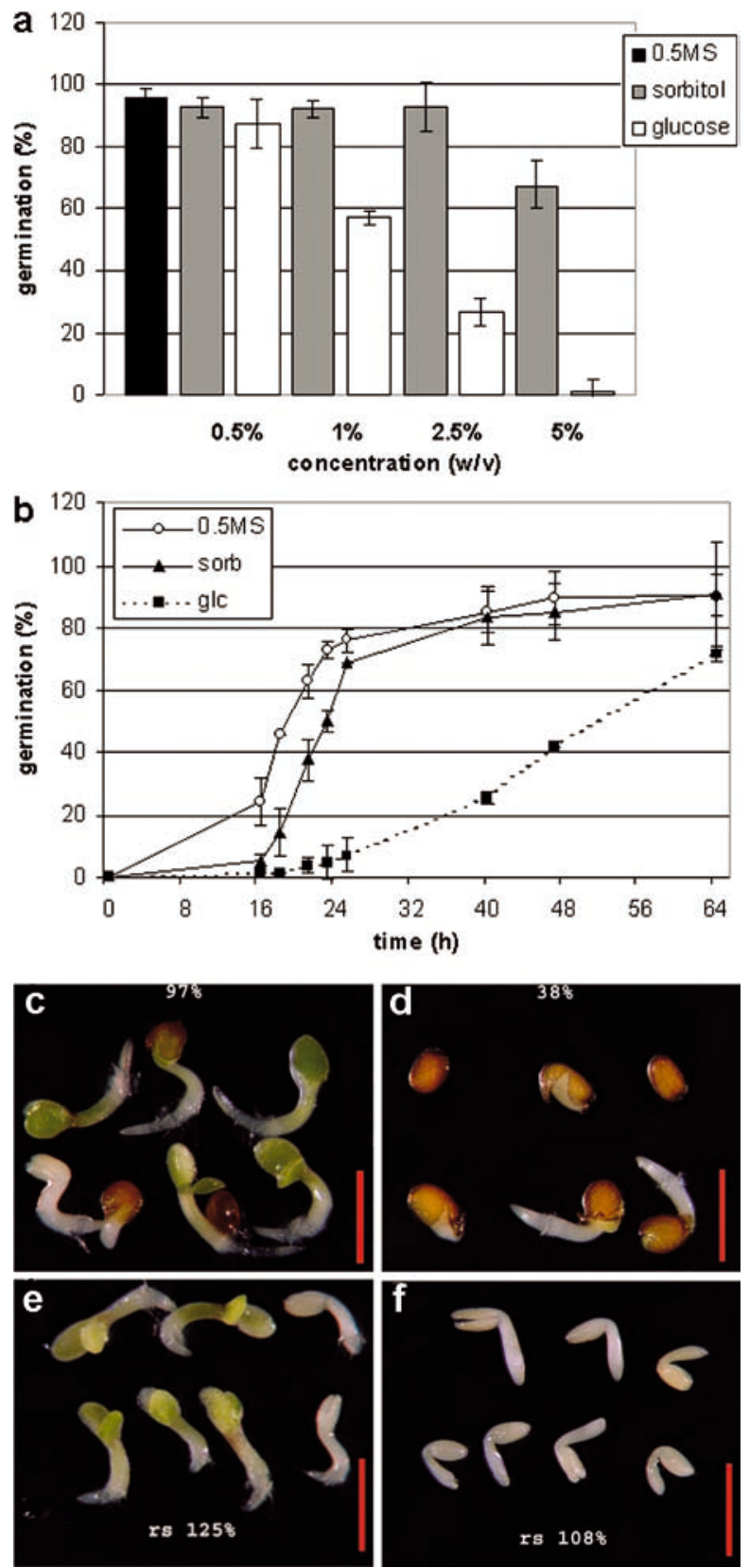

Fig. 1a-f Glucose delays germination of Arabidopsis thaliana seeds. a Germination inhibition of Ler seeds by increasing concentrations (w/v) of sorbitol and glucose scored $24 \mathrm{~h}$ poststratification. b Detailed time course of the germination response of Ler seeds on $0.5 \mathrm{MS}$, and on $0.5 \mathrm{MS}$ containing $2.5 \%$ sorbitol or $2.5 \%$ glucose. a and $\mathbf{b}$ show means \pm SE of duplicate experiments, with confidence of $99 \%$. Similar results were obtained in three independent experiments. c-f Germination of Col-0 seeds (c,d) and dissected embryos $(\mathbf{e}, \mathbf{f})$ on $0.5 \mathrm{MS}(\mathbf{c}, \mathbf{e})$ and on $2.5 \%$ glucose $(\mathbf{d}, \mathbf{f})$ at $38 \mathrm{~h}$ post-stratification. Germination percentage is indicted for the seeds. Also average relative sizes $(r s)$ of the dissected embryos are indicated. Representative embryos from seeds germinated on $2.5 \%$ glucose (as in d) were dissected and measured and the average was set to $100 \%$. Similar results were obtained in two other experiments using Col-0 seeds and Ler seeds, respectively

tested showed a delay in germination caused by glucose (data not shown). Although the range of germination inhibition differed among the different ecotypes, this 
indicates that the glucose-induced delay is a general phenomenon in Arabidopsis.

\section{Characterization of the sugar response}

Next we tested whether other sugars were able to trigger inhibition of seed germination. Germination was assayed on $0.5 \mathrm{MS}$ medium or on $0.5 \mathrm{MS}$ medium supplemented with chemicals that serve as osmotic controls (sorbitol, mannitol or $\mathrm{NaCl}$ ), or supplemented with different hexoses (glucose, galactose and 3-OMG) or disaccharides (sucrose, maltose or trehalose). All sugars tested were able to delay germination more efficiently than the osmotic controls although the effect of galactose, maltose and trehalose was relatively small and rapidly disappeared. Sucrose, glucose and, in particular, the non-metabolically active glucose analog 3-OMG postponed germination more strongly (Fig. 2). Since sucrose is hydrolyzed to glucose and fructose these hydrolysis products might be responsible for the inhibition observed on sucrose. The observation that the non-metabolizable sugar analog 3-OMG is an effective inhibitor of germination suggests that HXK activity or further metabolism is not necessary to trigger the germination delay.

Sugar responses are influenced by nitrogen availability or carbon/nitrogen ratio (Martin et al. 2002). To investigate whether different nitrogen concentrations in the media affected the sugar-induced germination delay, Ler seeds were sown on $0.5 \mathrm{MS}$ or on $0.5 \mathrm{MS}+2.5 \%$ glucose with different $\mathrm{N}$ concentrations. Germination was scored daily for 4 days, and Fig. 3a shows that the germination curves on $0.5 \mathrm{MS}$ are similar for the different $\mathrm{N}$ concentrations. This germination profile contrasts with the germination profile of glucose-treated seeds

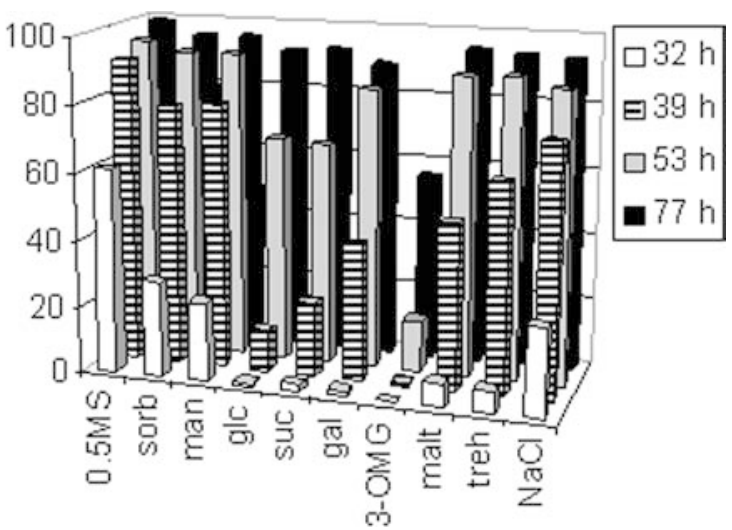

Fig. 2 Inhibition of Arabidopsis seed germination by different sugars. Ler seeds were germinated on $0.5 \mathrm{MS}$, and on $0.5 \mathrm{MS}$ containing $84 \mathrm{mM}(=1.5 \%$ glucose $)$ sugar or $42 \mathrm{mM} \mathrm{NaCl}$. Germination was scored in time (indicated on the right). sorb Sorbitol, man mannitol, suc sucrose, gal galactose, 3-OMG 3-Omethylglucose, malt maltose, treh trehalose. Germination in the presence of the different sugars was tested at least four times except for trehalose and $\mathrm{NaCl}$, which were tested twice
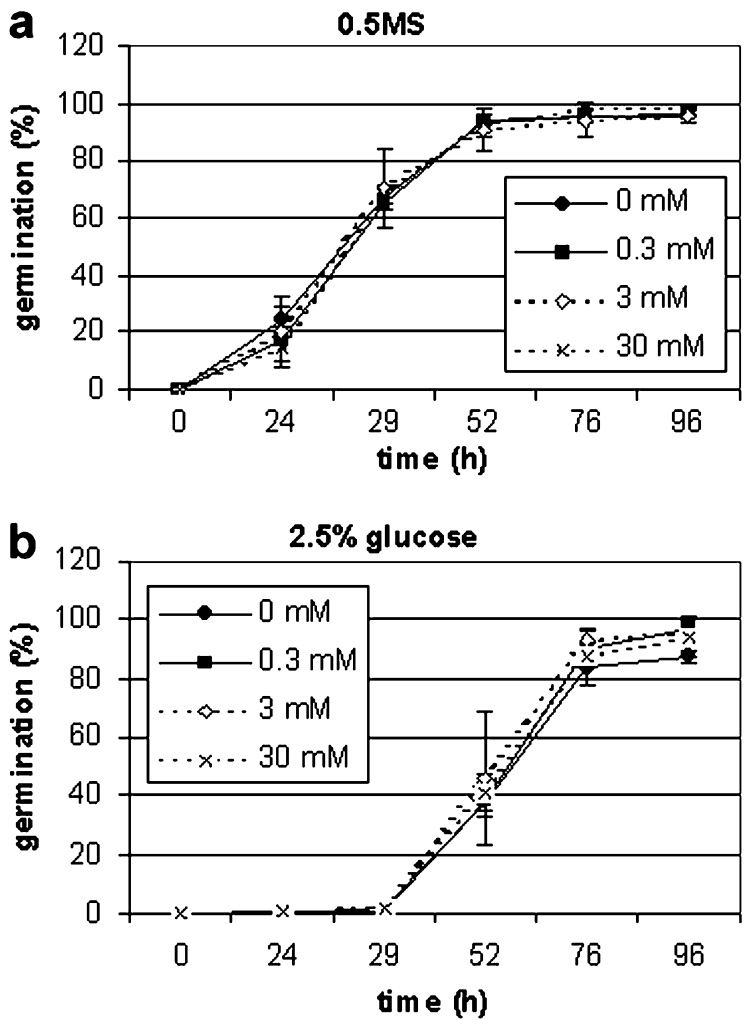

Fig. 3a,b Effect of nitrogen availability on glucose inhibition of Arabidopsis seed germination. Time course of germination of Ler seeds on $0.5 \mathrm{MS}$ (a), and on $0.5 \mathrm{MS}+2.5 \%$ glucose (b) in the presence of different nitrogen concentrations. For this experiment, $0.5 \mathrm{MS}$ media (without vitamins) were custom-made in the laboratory so we were able to manipulate the nitrogen concentration (as indicated in the figure). The concentration of $30 \mathrm{mM} \mathrm{N}$ corresponds to the amount of $\mathrm{N}$ in the $0.5 \mathrm{MS}$ medium. In the media with lower $\mathrm{N}$ concentration the ratio between nitrate and ammonium was maintained constant and $\mathrm{KCl}$ was added to obtain equal potassium ion concentrations. Data are means \pm SE (with confidence of $95 \%$ ) of a single experiment (performed in duplicate) and the same result was obtained in a second experiment

where a germination delay is observed. However, the response to the exogenous glucose is not altered by the different amounts of $\mathrm{N}$ in the germination media and is thus independent of nitrogen effects (Fig. 3b).

Fig. $1 \mathrm{~b}$ shows that $2.5 \%$ sorbitol has a mild osmotic effect, although a similar concentration of glucose severely inhibits germination. This inhibitory effect of $2.5 \%$ glucose could not be mimicked by a small amount of sugar $(0.5 \%)$ supplemented with sorbitol $(2 \%)$, indicating that that the inhibitory effect fully depends on glucose concentration and cannot be mimicked by osmotic stress (data not shown).

Hormones as possible signalling intermediates in glucose-mediated germination inhibition

From the above it is clear that glucose delays germination. To address the possible mechanism for the sugarmediated delay in germination the role of three plant hormones, namely ABA, GA and ethylene, was inves- 
tigated; GA and ethylene stimulate germination, whereas ABA is inhibitory (Koornneef and Karssen 1994; Bentsink and Koornneef 2002). We hypothesized that sugar might interact with hormone biosynthesis or signalling, which would explain the observed germination delay. A negative interference of sugar with a GAdependent signalling pathway has been described in barley embryos (Perata et al. 1997) and, interestingly, both ABA and ethylene were shown to be involved in sugar responses (e.g. Zhou et al. 1998; Huijser et al. 2000; Laby et al. 2000). Post-germination seedling growth is inhibited by high glucose concentrations mediated by ABA and $A B I 4$, and both factors control germination as well, and are therefore obvious candidates. To test this hypothesis, hormones and hormone inhibitors were applied to the germination media to uncover whether the germination behaviour of glucosetreated seeds was influenced. In addition, various mutants in ABA, ethylene and GA biosynthesis and signalling were screened in germination assays for their respective responses on sugar media.

The sugar effect is independent of GA or ethylene

GA is an important hormone for germination as shown by the observation that GA-deficient mutants do not germinate (Bentsink and Koornneef 2002). Germination can be induced in GA-deficient mutants by exogenous gibberellic acid $\left(\mathrm{GA}_{3}\right)$ application. Also, addition of ethylene [or its precursor 1-aminocyclopropane-1-carboxylic acid (ACC)] or BRs is able to overcome the germination arrest due to GA deficiency (Koornneef and Karssen 1994; Steber and McCourt 2001). Ler seeds were sown on $0.5 \mathrm{MS}$, or on $0.5 \mathrm{MS}$ containing $2.5 \%$ sorbitol or glucose. Glucose media contained $\mathrm{GA}_{3}$, ACC or BR in concentrations that are sufficient to overcome GA deficiency (Steber and McCourt 2001). These concentrations were tested in our conditions. $\mathrm{GA}_{3}(10 \mu \mathrm{M})$, ACC $(10 \mu \mathrm{M})$ and BR $(2.5 \mu \mathrm{M})$ were added to Ler and Col-0 seeds treated with $100 \mu \mathrm{M}$ paclobutrazol (PAC), a potent inhibitor of GA biosynthesis. All three hormones relieved the PAC-mediated germination inhibition (data not shown). Seeds sown on glucose media or glucose media supplemented with the different hormones show a similar germination curve (Fig. 4). Thus these germination-promoting hormones do not relieve the glucose inhibition, suggesting that biosynthesis of these hormones is not affected by glucose. To further investigate the role of GA and ethylene signalling pathways in glucose signalling during germination, GA (spy) and ethylene (etr1, ctr1, ein2, ein3) response mutants were tested on media containing $2.5 \%$ glucose and sorbitol. $S P Y$ encodes a putative $O$-linked $N$-acetyl-glucosamine transferase and is believed to act as a repressor of GA signalling (Olszewski et al. 2002). SPY is expressed during germination and spy mutants were identified in screens for mutants that were PAC insensitive during germination (Jacobsen and Olszewski 1993; Swain et al.

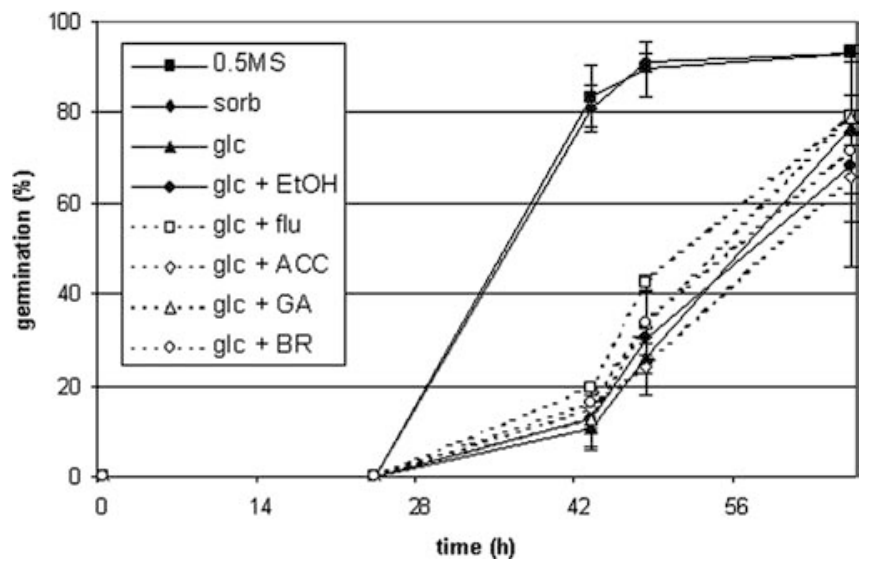

Fig. 4 Effect of hormone addition on glucose inhibition of Arabidopsis seed germination. Ler seeds were germinated on $0.5 \mathrm{MS}$, and on $0.5 \mathrm{MS}$ containing $2.5 \%$ sorbitol or $2.5 \%$ glucose, without or with addition of ethanol $(E t O H$, solvent), fluridone ( $f l u$, $10 \mu \mathrm{M}), \mathrm{ACC}(10 \mu \mathrm{M}), \mathrm{GA}_{3}(G A, 10 \mu \mathrm{M})$ or epibrassinolide $(B R$, $2.5 \mu \mathrm{M}$ ). Data are means \pm SE (with confidence of $95 \%$ ) of a single experiment (performed in duplicate), and similar results were obtained in two additional experiments

2002). The spy mutant does not show insensitivity to glucose during germination but in several independent experiments was somewhat more glucose sensitive compared to WT (Fig. 5a).

The ETRl gene encodes an ethylene receptor and acts upstream of CTR1, a member of the Raf family of Ser/ Thr protein kinases that negatively regulate ethylene signalling. Downstream of these two factors is positioned EIN2, which is an essential positive regulator of ethylene signalling during plant development. Further downstream, EIN3 acts as a transcriptional regulator (Roman et al. 1995; Wang et al. 2002). Mutations in these central regulators of ethylene signalling lead to ethylene insensitivity (etr1, ein2 and ein3) or to a constitutive ethylene response (ctrl) in plants. These mutants were tested for their response to glucose. The ctr 1-1 mutant is reproducibly somewhat more sensitive than WT (Fig. 5b), as observed for spy. Germination of Col-0 on glucose is not significantly different from that of the etr $1-1$ mutant (Fig. 5c). Interestingly, the ein 3-1 mutant behaves differently from the other ethyleneresponse mutants tested since it shows a partial insensitivity to glucose (Fig. 5d). This glucose insensitivity of ein3-1 was observed in three independent experiments. Apparently, EIN3 somehow interferes with glucose signalling during germination. The ein2-1 mutant was tested as well but its germination behaviour was complex, and in four experiments no clear reproducible results were obtained (data not shown).

Glucose does not affect ABA biosynthesis or signalling via $\mathrm{ABI} 4$ during germination

The important role of $\mathrm{ABA}$ in sugar responses and germination inhibition makes $\mathrm{ABA}$ and its signalling 
Fig. 5a-d Germination response on glucose of different Arabidopsis GA and ethylene signalling mutants.

Germination responses of WT (black lines and symbols) and mutant (dashed lines and open symbols) lines on $0.5 \mathrm{MS}$ (circles), and on $0.5 \mathrm{MS}$ containing $2.5 \%$ sorbitol (triangles) or $2.5 \%$ glucose (squares) are shown. Data are means of a single experiment (performed in duplicate). Error bars (SE, confidence of $95 \%$ ) are given only for the glucose data. Similar results were found in three independent experiments. Col-0 and etr 1 seeds were from a different batch than the Col- 0 , ctr 1 and ein3 seeds
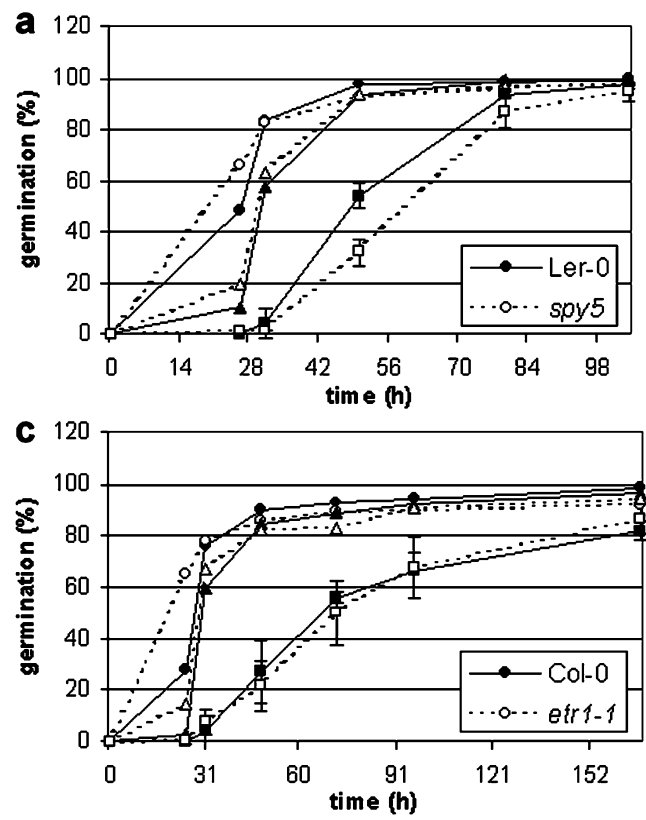
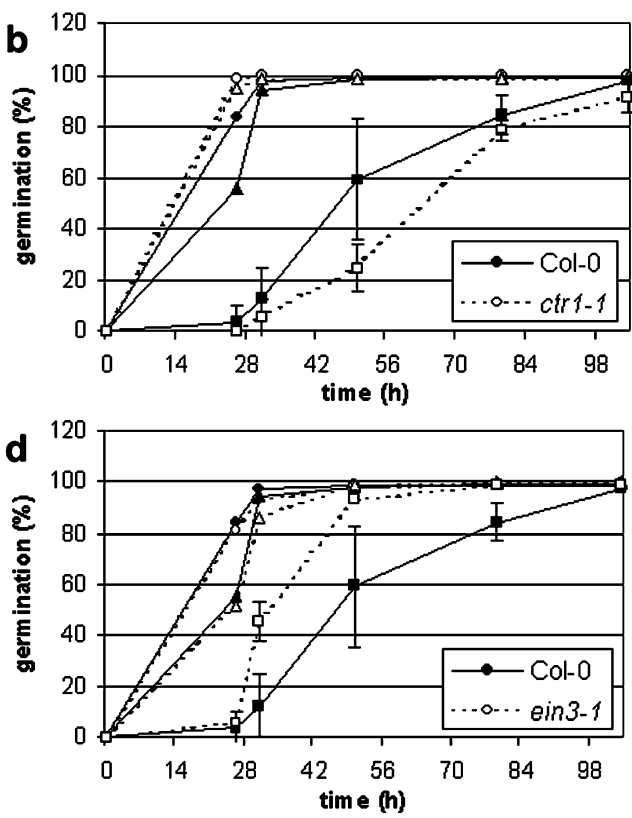

components potential candidates for transducing the inhibitory glucose signal during germination. Seeds were germinated on media supplemented with fluridone, an inhibitor of ABA biosynthesis. ABA biosynthesis is inhibited at low concentrations $(10 \mu \mathrm{M})$ of fluridone, which is already effective on imbibed seeds within hours following application (Grappin et al. 2000; Jullien et al. 2000). De-novo ABA biosynthesis was shown to be necessary to maintain dormancy in imbibed seeds (Jullien et al. 2000). Addition of fluridone does not suppress the glucose effect, indicating that an increase in ABA biosynthesis is not required (Fig. 4). In these experiments it is unlikely that fluridone uptake is blocked by the seed coat since the fluridone concentration used in this study $(10 \mu \mathrm{M})$ produced germinated embryos with a white/pink appearance as expected (data not shown). To further clarify the role of ABA biosynthesis the ABA biosynthesis mutant, $a b a 2$, was tested for its germination response. Germination of $a b a 2-1$ can be suppressed by glucose but it shows a moderate insensitivity to glucose compared to WT (Fig. 6a). Furthermore, seeds were sown on control media, ABA $(1 \mu \mathrm{M})$, glucose and glucose + ABA $(1 \mu \mathrm{M})$. The application of $1 \mu \mathrm{M}$ ABA slightly affected germination but combined with glucose it enhanced the germination inhibition compared to glucose alone (Fig. 6b). This suggests that ABA levels influence the glucose response during germination because reduced levels (aba2-1, Fig. 6a) lead to insensitivity and increased ABA levels (ABA application, Fig. 6b) promote the glucose-induced retardation of germination.

Other known ABA signalling mutants tested (abi2-1, abi5-1 and the ABA-, glucose- and salt-insensitive abi41 and abi4-2 (Koornneef et al. 1984; Finkelstein 1994; Quesada et al. 2000) showed a similar (or sometimes a somewhat enhanced) sensitivity to glucose in comparison with WT (Fig. 6c-f). Apparently, mutations in these
$A B I$ genes do not lead to glucose insensitivity during germination. These findings support the view that glucose does not act by increasing ABA biosynthesis and does not depend on $A B I 2, A B I 4$ and $A B I 5$ signalling.

\section{Discussion}

Seeds have mechanisms to prevent germination under adverse conditions such as osmotic stress (Carles et al. 2002). Osmotic and salt concentrations that inhibit WT germination are not inhibitory to abi4, abi5 and aba2 mutants (Quesada et al. 2000; Carles et al. 2002). However, such mutants, which are able to germinate on $175 \mathrm{mM} \mathrm{NaCl}$, die within 2 weeks, indicating that a proper response to potential environmental stresses is of vital importance for seedling survival, and that $\mathrm{ABA}$ and the ABI4 and ABI5 gene products play a key role in this process (Carles et al. 2002). Results presented in this paper suggest that glucose delays germination as well, although its physiological significance is obscure. This finding came as a surprise since ABA-induced germination arrest can be effectively reverted by sugar addition. The inhibitory effect of glucose with respect to germination is not due to osmotic stress since in these experiments sorbitol at equimolar concentrations is far less effective than glucose (Fig. 1a). Treatment with a low glucose concentration $(0.5 \%)$ mixed with sorbitol $(2.0 \%)$ cannot mimic the glucose $(2.5 \%)$-induced delay, suggesting that the glucose concentration itself is responsible independent of osmotic signals.

In the germination assay, several sugars were tested for their ability to inhibit seed germination. Glucose, sucrose and 3-OMG had pronounced effects. Interestingly, 3-OMG is efficiently transported but is hardly phosphorylated by HXK (Cortes et al. 2003). Its inhibitory effect suggests that HXK activity or further glucose 
Fig. 6a-f Effects of ABA, ABA biosynthesis and signalling mutants on the Arabidopsis germination response. a, c-f Germination responses of WT (black lines and symbols) and mutant (dashed lines and open symbols) lines on $0.5 \mathrm{MS}$ (circles), and on $0.5 \mathrm{MS}$ containing $2.5 \%$ sorbitol (triangles) or $2.5 \%$ glucose (squares) are shown. Data are means of a single experiment (performed in duplicate). Error bars (SE, confidence of $95 \%$ ) are given only for the glucose data. Similar results were found in at least three independent experiments. b Col-0 seeds were germinated on $0.5 \mathrm{MS}$ (control), and on $0.5 \mathrm{MS}$ containing ABA $(1 \mu \mathrm{M})$, glucose $(2.5 \%)$, or ABA $(1 \mu \mathrm{M})+$ glucose $(2.5 \%)$ $(A+G)$. Data are means $\pm \mathrm{SE}$ (with confidence of $95 \%$ ) of a single experiment (performed in duplicate). Similar results were obtained in a second independent experiment
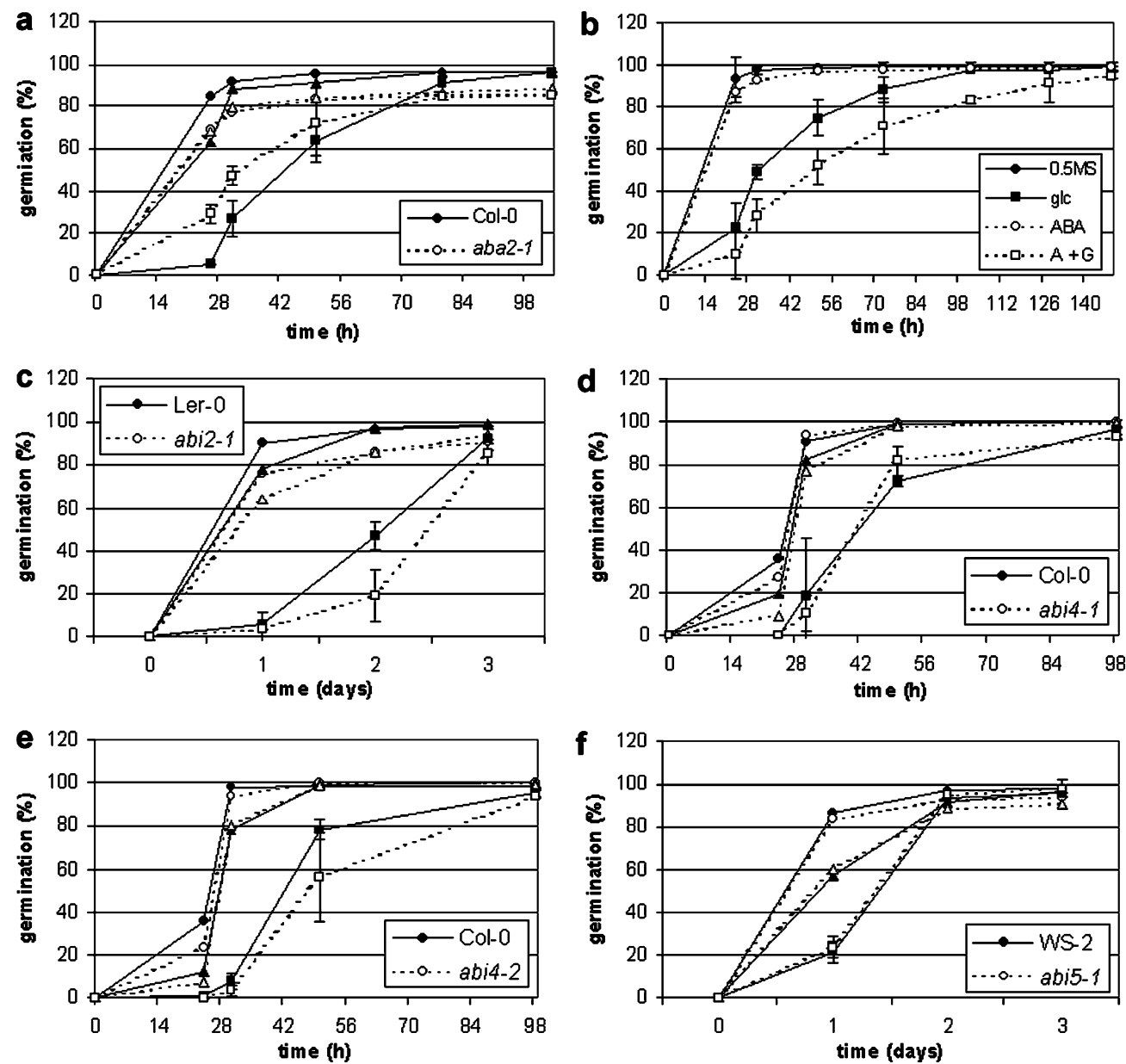

metabolism is not required to elicit the germination response. This conclusion is supported by the recent work of Price and co-workers (2003). Other sugars tested were less effective in causing a germination delay. The different levels of germination retardation by sugars might reflect the different ways, or efficiencies, in which these sugars are sensed, transported or metabolized. Martin et al. (2002) showed that high nitrogen concentrations suppress the sugar effect on early seedling growth and $C A B$ and $R B C S$ gene expression. These processes are proposed to be affected by HXK signalling (Sheen et al. 1999; Rolland et al. 2002). The observed sugar effect is not modulated by nitrogen availability, providing another argument that glucose inhibition of germination is an HXK-independent process. However, it is unclear to what extent Arabidopsis seeds are responsive to exogenous nitrogen availability. Nitrate application was found to stimulate Arabidopsis seed germination although it is not required (Bentsink and Koornneef 2002).

An earlier study showed that the glucose analog mannose is a very potent inhibitor of Arabidopsis seed germination and that even very low concentrations (5-10 $\mathrm{mM})$ are effective (Pego et al. 1999), yet the repressive mode of action of mannose is thus far unknown. It seems that the inhibition of germination described in this paper has a different mechanistic basis. In the mannose-insensitivity assay, $10 \mathrm{mM} 3-\mathrm{OMG}$, glucose and fructose were not inhibitory (Pego et al. 1999). In the germination assay described here, 3-OMG and glucose are only effective in higher concentrations. The effect of mannose could be counteracted by the addition of $60 \mathrm{mM}$ glucose, a concentration that is inhibitory in our assay. Furthermore, the mannose inhibition was proposed to depend on HXK activity. In our germination assay, 3-OMG inhibited germination as well, suggesting that HXK activity or glucose metabolism are not involved in inducing germination retardation. The sugar- and ABA-insensitive sun6/abi4 was identified as being mannose insensitive (Pego et al. 1999; Huijser et al. 2000). In the germination assay presented here the abi4 mutation did not result in an insensitive phenotype (Fig. 6d,e). These observations provide strong arguments for the notion that the germination-delaying effect described here differs from the mannose inhibition and involves a different signalling pathway. Furthermore, several sugar-insensitive mutants were isolated that were resistant to elevated sugar levels with respect to early seedling growth. This developmental block depends on several processes such as HXK activity, ABA biosynthesis and $A B I 4$ signalling (Rolland et al. 2002). However, for the glucose 
inhibition of germination described here, $H X K$ and $A B I 4$ do not seem necessary. Thus a glucose-sensitive signalling pathway is active during germination that is different from the $H X K 1 / \mathrm{ABA} / A B I 4$ signalling cascade active in early seedling growth.

The mechanism by which glucose represses the germination response is unknown. In yeast, sugar-sensing systems are known that operate at the cell membrane, e.g. SNF3 and RGT2 (Özcan et al. 1996). It is an intriguing possibility that this kind of signalling might delay seed germination. However, such mechanisms are thus far not described in plants. In Arabidopsis, 26 putative hexose transporters (HXT) have been proposed (Lalonde et al. 1999). Two out of these 26 HXTs contain extended central loops that might possibly be involved in signalling, although no evidence for such a proposed function is as yet available (Lalonde et al. 1999).

A non-enzymatic mechanism cannot be ruled out. As an aldehyde, glucose is chemically reactive. Biochemical interactions between reducing sugars and proteins, known as the Maillard reaction, lead to the formation of glycoproteins and advanced glycation end products (AGEs: heterogeneous group of structures formed as both cross-linking and non-cross-linking adducts on proteins; Nagaraj et al. 1996). The formation of these products is proposed to play an important role in protein aging. In the medical field, Maillard reaction products are known to be associated with complications in aging and diabetes (e.g. Nagaraj et al. 1996). In plants the Maillard reactions products are thought to influence seed colour during aging and are associated with seed viability loss depending on storage conditions (Taylor et al. 2000; Murthy et al. 2003). The germination delay observed could be due to such events but this is unlikely since the random process of glycoprotein formation would affect cellular processes in general. This does not easily explain why certain mutants are insensitive to glucose (like aba2, fus3, lecl and ein 3) and why low amounts of ABA in combination with glucose are able to promote the glucose retardation.

Complex interactions between sugar and hormones during germination

Germination is regulated by the growth potential of the embryo in the seed and the restrictive properties of the seed coat (Bentsink and Koornneef 2002). We demonstrate that removal of the seed coat leaves the embryos sensitive to inhibition by glucose, indicating that the effect of glucose is directed to the embryo and probably does not involve the seed coat. GA-deficient mutants fail to germinate but this problem can be overcome by releasing the embryos from their seed coats or by combining the $g a$ mutation with seed-coat mutants (Debeaujon and Koornneef 2000). Therefore, it was proposed that GA acts on the seed coat, possibly by promoting its weakening. Moreover, combining the $g a$ mutation with an ABA-deficient background enables seeds to germinate. In addition, some ABA-insensitive mutants (abi3 and abi4) are insensitive to inhibitors of GA biosynthesis (Koornneef and Karssen 1994; Laby et al. 2000; and our unpublished observations). The germination inhibition by glucose might involve negative interference with GA biosynthesis or signalling. However, such a model seems unlikely in our case since: (i) the spy mutation does not relieve the glucose delay, (ii) addition of GA, ACC or BR does not relieve glucose inhibition, (iii) abi4 and WT are similarly sensitive to sugar. The data presented fail to support an antagonistic role for ethylene in germination inhibition by glucose. Neither the addition of ACC nor a constitutive ethylene response caused by ctr $1-1$ relieves the glucose-induced germination delay. The ethylene mutant etrl seems slightly more sensitive to glucose but, remarkably, ein 3 shows a decreased sensitivity to glucose (Fig. 5d). EIN3 encodes a DNA-binding protein that is involved in ethylene signalling (for review, see Wang et al. 2002). It is nuclear-localized and binds the primary ethylene-response element (Solano et al. 1998). Possibly, EIN3 is regulated by other signalling cascades as well because ACC application and ctrl do not affect the glucose-induced delay. The ctrl mutant is sugar insensitive for the sugar-induced block of early seedling growth, and etr 1 and ein 3 are glucose oversensitive (Gibson et al. 2001; León and Sheen 2003). In contrast, during germination the ctr 1 mutation does not lead to a glucose-insensitive phenotype, etr 1 does not show a significantly enhanced sensitivity during germination and ein 3 is glucose insensitive instead of glucose oversensitive. Thus, these results obtained with different ethylene signalling mutants also support the conclusion that the glucose inhibition of germination is distinct from the inhibition of early seedling growth and involves a separate signalling pathway.

In contrast to ethylene and GA, ABA seems to be involved in the regulation of glucose inhibition of germination because ABA levels may determine the severity of the glucose response. The ABA biosynthetic mutant aba2-1 shows a decreased sensitivity with respect to glucose inhibition of germination (Fig. 6a). This is supported by results obtained by Ullah and co-workers (2002) who show that fluridone pre-treatment leads to glucose insensitivity during germination. It has been found that ABA levels drop rapidly in seeds after fluridone treatment (Grappin et al. 2000; Jullien et al. 2000). Thus it remains unclear whether the effect of fluridone pre-treatment on glucose inhibition is due to a reduced ABA content or to the inability to increase ABA levels. However, in our experiments no effects of fluridone were observed when it was applied together with glucose. In this case, seeds were exposed to both chemicals simultaneously and the glucose response might be faster than the effect of fluridone in lowering ABA levels. Moreover, the application of $6 \%$ glucose did not induce increased ABA levels (Ullah et al. 2002). Possibly, this inhibitory pathway is not regulated by increasing ABA biosynthesis, but is affected by the ABA levels present in the 
seed, which might affect the sensitivity to the glucose inhibitory pathway. This is supported by the fact that low ABA concentrations in combination with glucose enhance the glucose response (Fig. 6b) and that decreased ABA levels lead to glucose insensitivity (Fig. 6a; Ullah et al. 2002; Price et al. 2003). However, it cannot be ruled out that $\mathrm{ABA}$ is more directly involved, e.g. glucose might affect ABA stability or signalling.

The data suggest that ABA is able to affect glucose inhibition of germination but, interestingly, the abi2, abi4 and abi5 mutants show WT sensitivity to glucose with respect to germination inhibition. This is supported by recent data from Brocard-Gifford et al. (2003) and Price et al. (2003), which also showed that during germination the response of both abi4 and abi5 to glucose is similar to that of WT. The proposed involvement of ABA in affecting the glucose inhibition does not depend on $A B I 2, A B I 4$ or $A B I 5$ function. Neither is it antagonized by ACC or GA application or constitutive ethylene or GA responses caused by the ctrl and spy mutations, respectively. These observations are in contrast to findings that $\mathrm{ABI}$ gene products are required for ABA-mediated inhibition of seed germination (Koornneef et al. 1984; Finkelstein 1994), and that both ethylene and GA are able to antagonize ABA action (Beaudoin et al. 2000; Ghassemian et al. 2000; Bentsink and Koornneef 2002). It has been suggested that ABA might affect germination by more than one pathway (as discussed by Pritchard et al. 2002). Possibly, these different actions are separated spatially or temporally during germination and this question will be addressed in future work. Recently it was shown that fus 3 (and to a minor extent lec 1 ) shows insensitivity to glucose-induced germination inhibition (Brocard-Gifford et al. 2003). Interestingly, fus 3 mutant seeds have a WT response to ABA and posses WT ABA levels 15 days after pollination (Brocard-Gifford et al. 2003; Nambara et al. 2000) This indicates that normal seed development and, in particular, FUS3 function are important for proper responses to glucose during germination. In this light, it would be of interest to test a severe abi3 mutant for glucose inhibition of germination because this mutant has seed developmental defects as well (Ooms et al. 1993; Nambara et al. 2000).

In conclusion, metabolizable sugars can stimulate germination in repressive situations (e.g. ABA or mannose treatment, Pego et al. 1999; Finkelstein and Lynch 2000) but the data presented here show that sugars are, even at low concentrations, inhibitors of germination as well. Sugars negatively affect plant embryo growth. This inhibition is distinct from the earlier described mannose inhibition of germination (Pego et al. 1999) and the $H X K / \mathrm{ABA} / A B I 4$ signalling cascade. We suggest that this delay in germination represents a separate signalling branch in the regulation of germination and is independent of the ABI genes tested. Mutant selection would be an appropriate approach to identify components of this unknown sugar-dependent signalling pathway in plants and/or additional factors that control seed germination.
Acknowledgements The authors greatly acknowledge B. Verhagen (Phytopathology, University of Utrecht), Dr. T. Peeters (Ecophysiology, University of Utrecht), Dr. J.L. Micol (Miguel Hernandez University, Alicante, Spain) and the Nottingham Arabidopsis Stock Centre (NASC) for providing seeds, Frits Kindt for help with photography and the Imageprocessing \& Design department (Faculty of Biology, University of Utrecht) for help preparing the figures. This research was supported by the Earth and Life Sciences Foundation subsidized by The Netherlands Organization for Scientific Research.

\section{References}

Arenas-Huertero F, Arroyo A, Zhou L, Sheen J, Leon P (2000) Analysis of Arabidopsis glucose insensitive mutants, gin5 and gin6, reveals a central role of the plant hormone ABA in the regulation of plant vegetative development by sugar. Genes Dev 14:2085-2096

Beaudoin N, Serizet C, Gosti F, Giraudat J (2000) Interactions between abscisic acid and ethylene signaling cascades. Plant Cell 12:1103-1116

Bentsink L, Koornneef M (2002) Seed dormancy and germination. In: Somerville CR, Meyerowitz EM (eds) The Arabidopsis book. http://www.aspb.org/publications/arabidopsis/, American Society of Plant Biologists, Rockville, MD. DOI 10.1199/tab.0050

Brocard-Gifford IM, Lynch TJ, Finkelstein RR (2003) Regulatory networks in seeds integrating developmental, abscisic acid, sugar, and light signaling. Plant Physiol 131:78-92

Carles C, Bies-Etheve N, Aspart L, Leon-Kloosterziel KM, Koornneef M, Echeverria M, Delseny M (2003) Regulation of Arabidopsis thaliana Em genes: role of ABI5. Plant J 30:373-383

Cortes S, Gromova M, Evrard A, Roby C, Heyraud A, Rolin DB, Raymond P, Brouquisse RM (2003) In plants, 3-O-methylglucose is phosphorylated by hexokinase but not perceived as a sugar. Plant Physiol 131:824-837

Debeaujon I, Koornneef M (2000) Gibberellin requirement for Arabidopsis seed germination is determined both by testa characteristics and embryonic abscisic acid. Plant Physiol 122:415-424

Finkelstein RR (1994) Mutations at two new Arabidopsis ABA response loci are similar to the abi3 mutations. Plant J 5:765-771

Finkelstein RR, Lynch TJ (2000) Abscisic acid inhibition of radicle emergence but not seedling growth is suppressed by sugars. Plant Physiol 122:1179-1186

Garciarrubio A, Legaria JP, Covarrubias AA (1997) Abscisic acid inhibits germination of mature Arabidopsis seeds by limiting the availability of energy and nutrients. Planta 203:182-187

Gazzarrini S, McCourt P (2001) Genetic interactions between ABA, ethylene and sugar signaling pathways. Curr Opin Plant Biol 4:387-391

Ghassemian M, Nambara E, Cutler S, Kawaide H, Kamiya Y, McCourt P (2000) Regulation of abscisic acid signaling by the ethylene response pathway in Arabidopsis. Plant Cell 12:11171126

Gibson SI (2000) Plant sugar-response pathways. Part of a complex regulatory web. Plant Physiol 124:1532-1539

Gibson SI, Laby RJ, Kim D (2001) The sugar-insensitive1 (sis1) mutant of Arabidopsis is allelic to ctrl. Biochem Biophys Res Commun 280:196-203

Grappin P, Bouinot D, Sotta B, Miginiac E, Jullien M (2000) Control of seed dormancy in Nicotiana plumbaginifolia: postimbibition abscisic acid synthesis imposes dormancy maintenance. Planta 210:279-285

Huijser C, Kortstee A, Pego J, Weisbeek P, Wisman E, Smeekens S (2000) The Arabidopsis SUCROSE UNCOUPLED-6 gene is identical to ABSCISIC ACID INSENSITIVE-4: involvement of abscisic acid in sugar responses. Plant J 23:577-585

Jacobsen SE, Olszewski NE (1993) Mutations at the SPINDLY locus of Arabidopsis alter gibberellin signal transduction. Plant Cell 5:887-896 
Jullien M, Bouinot D, Ali-Rachedi S, Sotta B, Grappin P (2000) Abscisic acid control of seed dormancy expression in icotiana plumbaginifolia and Arabidopsis thaliana. In: Viemont J-D, Crabbe $\mathbf{J}$ (eds) Dormancy in plants from whole plant behaviour to cellular control. CABI, Wallingford, UK, pp 195-210

Koch KE (1996) Carbohydrate-modulated gene expression in plants. Annu Rev Plant Biol 47:509-540

Koornneef M, Karssen CM (1994) Seed dormancy and germination. In: Meyerowitz EM, Somerville CR (eds) Arabidopsis. Cold Spring Harbor Laboratory Press, Cold Spring Harbor, NY, pp 313-334

Koornneef M, Jorna ML, Brinkhorst-Van der Swan DLC, Karssen CM (1982) The isolation of abscisic acid (ABA) deficient mutants by selection of induced revertants in non-germinating gibberellin sensitive lines of Arabidopsis thaliana (L.) Heynh. Theor Appl Genet 61:385-393

Koornneef M, Reuling G, Karssen CM (1984) The isolation and characterisation of abscisic acid insensitive mutants of Arabidopsis thaliana. Physiol Plant 61:377-383

Laby R, Kincaid M, Kim D, Gibson S (2000) The Arabidopsis sugar-insensitive mutants sis4 and sis 5 are defective in abscisic acid synthesis and response. Plant J 23:587-596

Lalonde S, Boles E, Hellmann H, Barker L, Patrick JW, Frommer WB, Ward JM (1999) The dual function of sugar carriers: transport and sugar sensing. Plant Cell 11:707-726

León P, Sheen J (2003) Sugar and hormone connections. Trends Plant Sci 8:110-116

Martin T, Oswald O, Graham IA (2002) Arabidopsis seedling growth, storage lipid mobilization, and photosynthetic gene expression are regulated by carbon:nitrogen availability. Plant Physiol 128:472-481

Moore B, Zhou L, Rolland F, Hall Q, Cheng W, Liu Y, Hwang I, Jones T, Sheen J (2003) Role of the Arabidopsis glucose sensor HXK1 in nutrient, light, and hormonal signaling. Science 300:332-336

Murthy UMN, Kumar PP, Sun WQ (2003) Mechanisms of seed ageing under different storage conditions for Vigna radiata $(\mathrm{L}$. Wilczek: lipid peroxidation, sugar hydrolysis, Maillard reactions and their relationship to glass state transition. J Exp Bot 54:1057-1067

Nagaraj RH, Shipanova IN, Faust FM (1996) Protein cross-linking by the Maillard reaction. J Biol Chem 271:19338-19345

Nambara E, Hayama R, Tsuchiya Y, Nishimura M, Kawaide H, Kamiya Y, Naito S (2000) The role of ABI3 and FUS3 loci in Arabidopsis thaliana on phase transition from late embryo development to germination. Dev Biol 220:412-423

Olszewski N, Sun TP, Gubler F (2002) Gibberellin signaling: biosynthesis, catabolism, and response pathways. Plant Cell [Suppl] 14:61-80

Ooms JJJ, Léon-Kloosterziel KM, Bartels D, Koornneef M, Karssen CM (1993) Acquisition of desiccation tolerance and longevity in seeds of Arabidopsis thaliana. Plant Physiol 102:1185-1191

Özcan S, Dover J, Rosenwald AG, Wolfl S, Johnston M (1996) Two glucose transporters in Saccharomyces cerevisiae are glucose sensors that generate a signal for induction of gene expression. Proc Natl Acad Sci USA 93:12428-12432

Pego JV, Weisbeek PJ, Smeekens SCM (1999) Mannose inhibits Arabidopsis germination via a hexokinase-mediated step. Plant Physiol 119:1017-1024

Perata P, Matsukura C, Vernieri P, Yamaguchi J (1997) Sugar repression of a gibberellin-dependent signaling pathway in barley embryos. Plant Cell 9:2197-2208

Price J, Li TC, Kang SG, Na JK, Yang JC (2003) Mechanisms of glucose signaling during germination of Arabidopsis. Plant Physiol 132:1424-1438

Pritchard SL, Charlton WL, Baker A, Graham IA (2002) Germination and storage reserve mobilisation are regulated independently in Arabidopsis. Plant J 31:639-647

Quesada V, Ponce MR, Micol JL (2000) Genetic analysis of salttolerant mutants in Arabidopsis thaliana. Genetics 154:421-436

Rolland F, Moore B, Sheen J (2002) Sugar sensing and signaling in plants. Plant Cell [Suppl] 14:185-205

Roman G, Lubarsky B, Kieber JJ, Rothenberg M, Ecker JR (1995) Genetic analysis of ethylene signal transduction in Arabidopsis thaliana: five novel mutant loci integrated into a stress response pathway. Genetics 139:1393-1409

Rook F, Corke F, Card R, Munz G, Smith C, Bevan MW (2001) Impaired sucrose-induction mutants reveal the modulation of sugar-induced starch biosynthetic gene expression by abscisic acid signalling. Plant $\mathbf{J}$ 26:421-433

Sheen J, Zhou L, Jang JC (1999) Sugars as signaling molecules. Curr Opin Plant Biol 2:410-418

Smeekens S (2000) Sugar-induced signal transduction in plants. Annu Rev Plant Biol 51:49-81

Solano R, Stepanova A, Chao Q, Ecker JR (1998) Nuclear events in ethylene signaling: a transcriptional cascade mediated by ETHYLENE-INSENSITIVE3 and ETHYLENE-RESPONSEFACTOR1. Genes Dev 12:3703-3714

Steber CM, McCourt P (2001) A role for brassinosteroids in germination in Arabidopsis. Plant Physiol 125:763-769

Swain SM, Tseng TS, Thornton TM, Gopalraj M, Olszewski NE (2002) SPINDLY is a nuclear-localized repressor of gibberellin signal transduction expressed throughout the plant. Plant Physiol 129:605-615

Taylor AG, Min T-G, Paine DH (2000) Maillard reactions cause browning in bean seed coat during ageing: inhibition by aminoguanidine. In: Black M, Bradford KJ, Vazquez-Ramos J (eds) Seed biology: advances and applications. CABI, Wallingford, UK, pp 189-195

To J, Reiter WD, Gibson S (2002) Mobilization of seed storage lipid by Arabidopsis seedlings is retarded in the presence of exogenous sugars. BMC Plant Biol 2:4

Ullah H, Chen JG, Wang S, Jones AM (2002) Role of a heterotrimeric $\mathrm{G}$ protein in regulation of Arabidopsis seed germination. Plant Physiol 129:897-907

Wang KLC, Li H, Ecker JR (2002) Ethylene biosynthesis and signaling networks. Plant Cell [Suppl] 14:131-151

Zhou L, Jang JC, Jones TL, Sheen J (1998) Glucose and ethylene signal transduction crosstalk revealed by an Arabidopsis glucoseinsensitive mutant. Proc Natl Acad Sci USA 95:10294-10299 